\title{
"ES COMO QUE SE ME FORMA UNA BURBUJA ACÁ": PROCEDIMIENTOS METAFÓRICOS EN LA INTERACCIÓN CARDIÓLOGO-PACIENTE
}

\author{
GUIOMAR ELENA CIAPUSCIO \\ Universidad de Buenos Aires - Facultad de Filosofía y Letras \\ Instituto de Filología y Literaturas Hispánicas "Dr. Amado Alonso" \\ CONICET - Buenos Aires, Argentina
}

\section{RESUMEN}

Sobre la base de la teoría conceptual de la metáfora y desde la perspectiva del análisis de la conversación, este trabajo se enfoca en las metáforas conceptuales, las expresiones metafóricas y el trabajo de formulación que realizan médicos y pacientes para comunicar distintos aspectos de las crisis cardíacas. Las principales preguntas que articulan este trabajo son las siguientes: ¿cómo describen y reconstruyen los interactuantes las crisis cardíacas y los eventos asociados a ellas? ¿Qué papel y relevancia tienen en esas tareas conversacionales los procedimientos metafóricos? De acuerdo con los papeles discursivos, ¿es posible identificar especificidades en la selección del tipo de metáforas, en los procedimientos y recursos de formulación escogidos, y en las funciones que adquieren en los intercambios? El análisis se basa en un corpus de conversaciones entre cardiólogos y pacientes, realizadas en distintas instituciones de salud de Argentina.

PALABRAS CLAVE: comunicación médico-paciente, metáforas, reformulación, metadiscurso, metáforas del dolor.

\section{AbSTRACT}

On the basis of conceptual metaphor theory and from the perspective of conversation analysis, this paper focuses on conceptual metaphors, metaphorical expressions, and the formulation efforts carried out by doctors and patients to communicate different aspects of cardiac crises. The main questions articulated in this study are: How do interactants describe and reconstruct cardiac crises and associated events? What role and relevance do metaphorical procedures have in those conversational tasks? According to the discursive roles, is it possible to identify specificities in the selection of metaphor type, in the chosen procedures and formulation resources, and in the functions metaphors acquire in the exchanges? 
The analysis is based on a corpus of conversations among cardiologists and patients that took place in different healthcare services institutions in Argentina.

KEYWORDS: doctor-patient communication, metaphors, reformulation, metadiscourse, pain metaphors.

\section{INTRODUCCIÓN}

Este trabajo forma parte de un proyecto de investigación más amplio ${ }^{1}$, cuyo objetivo es estudiar los procedimientos y recursos de formulación que emplean pacientes y médicos para comunicar temáticas vinculadas a la enfermedad. En las interacciones entre médicos y pacientes las asimetrías de tipos de conocimiento (experto versus vivencial) y de afectación personal son notables. Comunicar conocimiento especializado, por parte de los médicos, y transmitir la experiencia subjetiva de la enfermedad y los dolores que ésta causa, por parte de los pacientes, constituyen tareas conversacionales complejas. Por estos motivos, distintos procedimientos lingüísticodiscursivos son aprovechados de manera intensa en estos intercambios: me refiero a los procedimientos de reformulación y calificación metadiscursiva (Gülich y Kotschi, 1995) y a los procedimientos metafóricos o de ilustración (Brünner y Gülich, 2002; Gülich, 2003; Ciapuscio, 2003, 2007).

El interés de este artículo radica en comprender y describir cómo los interlocutores reconstruyen y relatan las crisis cardíacas, poniendo el foco en los procedimientos y recursos metafóricos de que se valen y en el trabajo de formulación que realizan para llevar adelante las tareas conversacionales. Se trata, por tanto, de un análisis que observa y se detiene en los procedimientos metafóricos considerados en un contexto de uso real, es decir, tal como los emplean hablantes particulares en interacciones orales auténticas. Distintos autores han destacado la necesidad de complementar los avances de la teoría conceptual de la metáfora con estudios empíricos basados en datos auténticos procedentes de distintas esferas discursivas. Este trabajo aspira a ser una contribución en ese sentido.

Las principales preguntas que articulan este trabajo son las siguientes: ¿Cómo describen y reconstruyen los interactuantes las crisis cardíacas y los eventos asociados a ellas? ¿Qué papel y relevancia tienen en esas tareas conversacionales los procedimientos metafóricos? Teniendo en cuenta los papeles de los interlocutores, ¿es posible identificar especificidades en la selección del tipo de metáforas, en los procedimientos y recursos de formulación escogidos, y en las funciones que adquieren en los intercambios?

1 "La comunicación de la medicina: procedimientos textuales y léxico-gramaticales de la explicación, el diagnóstico y la recomendación” (Agencia Nacional de Promoción Científica y Tecnológica, Argentina, Proyecto PICT 2120-2013). 


\section{ANTECEDENTES TEÓRICOS Y CONSIDERACIONES METODOLÓGICAS}

La teoría conceptual de la metáfora (formulada inicialmente por trabajos de Lakoff y Johnson, 1991 y 1999; también Reddy, 1979²) y ampliada en posteriores trabajos, que profundizan en su naturaleza neural (Lakoff, 2008 y 2014), sostiene que la metáfora es primariamente un mecanismo esencial del sistema cognitivo, que nos permite comprender un dominio en términos de otro, y secundariamente, un fenómeno lingüístico, gestual y visual: por ejemplo, concebimos la vida como un viaje, las ideas como alimentos, las teorías como edificios, etc.; es decir, los dominios abstractos son conceptualizados a través de dominios más concretos. Así, las emociones, como la ira, el amor o el dolor, son conceptualizadas y comunicadas sobre la base de percepciones sensoriales como el calor y la presión (Kövecses, 2000, 2008; Semino, 2010; Díaz Vera, 2012).

Las metáforas son constructos cognitivo-semióticos que se basan en una relación de similitud (o correspondencia) entre dominios experienciales o que crean tales relaciones de similitud. Frecuentemente las metáforas no ocurren aisladas, sino que conforman sistemas de metáforas, cuyos elementos se relacionan entre sí de manera sistemática. Las metáforas se realizan en el discurso a través de expresiones metafóricas, que son empleadas por los autores de la teoría conceptual como evidencia lingüística para validar la existencia de aquellos esquemas o correspondencias cognitivoneurales; por ejemplo, a la expresión "de repente sentí una puntada terrible en la cabeza” subyace la metáfora conceptual EL DOLOR ES UN INSTRUMENTO FILOSO (cfr. Ciapuscio, 2016). En la mayoría de los trabajos de la lingüística cognitiva, las expresiones lingüísticas metafóricas son extraídas y tratadas de manera aislada de su contexto de empleo, si bien en los últimos años se han intensificado las investigaciones basadas en corpus (por ejemplo, Deignan, 2008). Por lo demás, en la tradición de los estudios del discurso, existen numerosos antecedentes teórico-descriptivos sobre la metáfora en contexto, incluyendo la comunicación sobre la enfermedad, aunque dominantemente en géneros escritos, como la publicidad y la prensa (entre muchos otros, véanse los trabajos de Williams-Camus, 2009 y 2016).

Mi interés se dirige a interacciones orales, en las que los interlocutores llevan a cabo actividades lingüísticas en colaboración para describir y reconstruir sus episodios críticos. La producción oral se concibe aquí como un trabajo interaccional, que conlleva un esfuerzo significativo que deja huellas en la superficie textual (Antos, 1982). Sobre la base del estudio

\footnotetext{
${ }^{2}$ Una amplia discusión y una reseña crítica que pone en cuestión la autoría de Lakoff y Johnson sobre la concepción cognitiva de la metáfora puede encontrarse en el libro de Surmann (2005): el autor demuestra cómo esta idea de base tiene una larga historia en la reflexión intelectual europea.
} 
pormenorizado de esas huellas en amplios corpus de datos orales, Gülich y Kotschi (1995) caracterizaron distintos procedimientos de formulación del discurso, que se revelan como regulares y "metódicos": por ejemplo, las reformulaciones (la vuelta atrás en el discurso para ampliar, corregir, precisar lo dicho) y los comentarios metadiscursivos, es decir, las actividades lingüísticas con las que los interlocutores comentan y valoran expresiones del discurso propio o del discurso ajeno. Las dificultades de formulación que supone toda interacción -y más aún, en contextos especializados, como la salud o las ciencias- explican el empleo asiduo de esos procedimientos y, también, de los procedimientos de verbalización metafóricos (Brünner y Gülich, 2002; Gülich, 2003; Ciapuscio, 2003), que permiten a los especialistas transmitir conocimiento especializado, y a los legos, comunicar percepciones corporales y vivencias personales de su enfermedad.

Para responder de manera preliminar a las preguntas formuladas en la introducción, estudio un corpus de trabajo formado por seis entrevistas entre cardiólogos y pacientes cardíacos, tomadas en el hospital público "El Cruce" situado en una localidad de Florencio Varela, y ocho entrevistas realizadas en dos clínicas privadas de la ciudad de Buenos Aires ${ }^{3}$. Las conversaciones, en el primer caso, tienen lugar pocos días después de ocurrida y superada la crisis cardíaca y son denominadas "entrevistas de ingreso"; en los dos últimos, durante el tratamiento y la rehabilitación. El análisis que presento a continuación es de orden inductivo y ejemplar, y sigue la orientación teórica del análisis conversacional: las contribuciones de los hablantes se analizan de manera secuencial, respetando el orden de la interacción, y se concentra en las formulaciones que van realizando en colaboración interactiva, a fin de lograr las metas discursivas compartidas, esto es, reconstruir el evento crítico, obtener y elaborar información vital para el diagnóstico y el tratamiento, una vez superada la crisis. El foco del análisis, de acuerdo con las preguntas iniciales, se coloca en las narraciones y descripciones de las crisis cardíacas y del dolor experimentado.

\section{ANÁlisis}

\subsection{Narrar la crisis cardiaca}

Las personas afectadas, al ser interrogadas por los médicos sobre sus eventos críticos (mayormente infartos y los síntomas concomitantes), recurren

\footnotetext{
${ }^{3}$ El protocolo de la investigación y la toma de datos fueron autorizados por el Comité de Ética de Investigación del Hospital y, en todos los casos, contaron con el consentimiento informado de los pacientes.
} 
de manera dominante a la narración como método conversacional para reconstruir lingüística y comunicativamente los sucesos pasados. Mediante este método reconstructivo (Bergmann y Luckmann, 1995), los pacientes, apoyados por las preguntas y comentarios de los médicos, evocan y presentan la historia de la enfermedad, los sucesos previos, las crisis y sus consecuencias, y para ello apelan a distintas técnicas narrativas; esencialmente, la narración iterativa (reconstrucción de sucesos recurrentes que se presentan como típicos), la narración episódica (la reconstrucción de un episodio singular, que se delimita claramente del resto de las acciones y eventos) y la representación escénica (escenificaciones), técnica mediante la cual lo pasado se coloca en la escena presente, los hechos y sucesos se narran como si estuvieran ocurriendo ante los ojos, es decir, la narración se vuelve drama (Gülich, 2007). En el marco de estas narraciones, las metáforas toman un protagonismo notable.

A modo de introducción, presento el siguiente ejemplo ${ }^{4}$, en que puede observarse la reconstrucción parcial de un infarto, ocurrido quince días antes de la conversación. El intercambio muestra el trabajo conversacional del paciente $(\mathrm{P})$, un familiar (F) y el médico $(\mathrm{M})$; los tres colaboran de distinto modo con el avance de la conversación: el paciente relata al médico el episodio de su ataque; su mujer (F), como testigo participante del evento crítico, aporta detalles y precisiones; el médico, por su parte, formula preguntas y comentarios dirigidos especialmente a obtener información específica sobre las circunstancias, la cualidad e intensidad del dolor.

Inmediatamente antes del fragmento citado, el paciente ha referido sensaciones y síntomas vividos en las horas previas al ataque, como falta de apetito, cansancio, desubicación espacial y confusión, situación que describe como "abombamiento".

(1) CRU $1^{5}$

1 P: 0 sea estaba como abombAdo y me fui a CAsa.. y guardé el AUto en casa y:

2 P: le comento a mi mujer.. que yo estaba como abombado y me dice no será. algo que te debe

3 P: haber paSAdo... y no f/

4 M: $\quad$ y aHÍ no fue a ningún hospiTA:L ninguna salita ahí no consultó’

5 P: no=no ningún lado no porque no no porque me senté

$6 \mathrm{M}: \quad$ no se tomó la presión tampoco. nadá

7 P: no= no. me senté en la mesa comimos algo qué se yo.

\footnotetext{
${ }^{4}$ Las convenciones de transcripción se encuentran al final del trabajo.

${ }^{5}$ CRU significa que los datos proceden del hospital "El Cruce" (las entrevistas se identifican numéricamente); los datos correspondientes a la clínica de diagnóstico y tratamiento, y a la clínica de rehabilitación se rotulan con CLT y CLR, respectivamente.
} 
8 P: terminé de comEr.. me voy a acostar no tengo ganas muchas ganas de comER

$9 \mathrm{~F}: \quad \quad \mathrm{y}$ dijiste no tengo ganas de comer

10 P: y me fui a acostar, y a las trEs de la mañana cuando me levanté me levanté con

$11 \mathrm{~F}: \quad$ estoy cansado

12 P: dolor de pecho y ahí NO no yo me levanto porque me levanto a esa hora

14 M: bien. [lo despertó el dolor o se levantó usted'

15 P: me sent/ me senté en el comedor. e: trabajo con mi Auto. de remIS pero me voy a la

$16 \mathrm{M}$ : bien. usted de qué trabaja'

17 P: caPITAL. entonces yo me voy a las cuatro cuatro y pico de la mañana salgo

$18 \mathrm{M}$ : está bien. entonces se despert/

19 M: sedesperTó y a lo largo le surge un dolor

20 P: me desperTÉ estaba tomando mate cocido no. estaba tomando mate cocido

21 P: sentado prendí la televisión. me preparé el/ el mate coCIdo TOdo. porque ella me deja todo

22 P: prepaRAdo viste' pero. calentar el Agua el microondas y en un tIro.. y en un tiro me: siento

23 P: un dolor en el pecho tremendo. entonces trato de abrir la puerta de:: de mi casa hacia el

24 P: jarDÍN. <rápido > y me voy para afuera porque me doy cuenta que no puedo respirar+.. abro

25 P: la canIlla de:: de afuera del jardín. y meto la cabEza abajo de la caNIlla. como para tomar

26 P: frEsco. Aire viste'. por el abombaMIENto que tenía.. era mUY agudo

27 M: [y el dolOR cómo era'eral'

28 M: [qué sentía usted'

29 P: era era presión era como que me agaRRAban con una mano un pedazo de carne acá

30 M: ajá $\quad$ y el dolor se quedaba ahí en el PEcho o se iba para

31 P: dentro asi <ruido con la boca de desgarro> sísínO se me iba

32 M: la espalda los bRAzos el cuello ahí y le faltaba el aire

33 P: nono para/ no aHÍ lo tenía ahí lo tenía ahí lo tenía y yo

34 P: me pegué y me empecÉ a faltar el aire. entonces le digo a mi mujer le digo:: da traé las llaves

35 P: del auto que nos vamos sí sí. cuando le digo

36 F: no despuÉS te empezó a doler el dorso. izquierdo,

37 P: traéme las llaves del Auto que nos vamos porque me parece que eSto es un infaRto le dije

El fragmento permite introducir los aspectos más relevantes considerados en la investigación, a saber: el recurso a diferentes técnicas narrativas, el trabajo colaborativo de los interactuantes para el desarrollo del tema, los procedimientos y recursos para transmitir la experiencia del dolor cardíaco, y, el fenómeno central para este trabajo: el recurso a la metáfora. 
Para reconstruir el evento crítico, ocurrido en el pasado reciente, $\mathrm{P}$ recurre a la narración episódica como estrategia narrativa dominante; en ese marco, apela en determinados momentos también a la presentación escénica, trayendo al presente la representación dramática de sucesos determinados, que perduran en su memoria de manera vívida. En las primeras líneas (1-10), P introduce las circunstancias que enmarcan lo sucedido: su estado de confusión al llegar a su casa, el diálogo con su mujer (que reproduce bajo la forma de una teatralización o presentación escénica: véase el empleo del tiempo presente y de discurso directo: "y me dice no será algo que ...”); luego, las acciones previas al ataque ("me senté a en la mesa, comimos algo (...) y me fui a acostar"). En ese pasaje pueden observarse, además, las colaboraciones de $\mathrm{F}$ para completar el relato, que en rigor consiste en una co-construcción (obsérvense las citas directas del discurso original de $\mathrm{P}$, que carecen de introductor alguno (en la línea 11, "estoy cansado"). Por último, en las líneas 4 y 6 pueden verse las intervenciones del médico, generalmente en forma de preguntas dirigidas a obtener información específica sobre las medidas terapéuticas eventualmente tomadas.

En la línea 10 comienza el relato del episodio central, el evento crítico, que se localiza temporalmente de manera precisa en pretérito indefinido y con una determinación horaria, delimitándolo así de manera clara de los sucesos previos y siguientes ("y a las tres de la mañana cuando me levanté me levanté con dolor de pecho..."). Luego de un intercambio secundario sobre el horario (que llama la atención del médico), en la línea 21 P retoma su narración -que se extiende hasta la línea 37. Como es muy frecuente en las narraciones sobre crisis cardíacas, el $\mathrm{P}$ se refiere al ataque como algo "repentino" (en línea 22 puede observarse la reiteración de la expresión "en un tiro", que ha de interpretarse como "muy velozmente') ${ }^{7}$ : "en un tIro.. y en un tiro me:. siento un dolor en el pecho tremendo. entonces trato de abrir la puerta de:: de mi casa (...) me voy para afuera porque me doy cuenta que no puedo respirar". Un aspecto relevante para subrayar es el notorio cambio de los tiempos de los verbos que realiza el narrador: las acciones dramáticas del evento principal se formulan en presente histórico; de este modo, se presentan como si estuvieran ocurriendo en el momento de la enunciación, lo cual permite la expresión enfática y vívida de lo experimentado.

${ }^{6}$ El carácter repentino del infarto coincide con lo hallado por Brünner y Gülich (2002) en el corpus en lengua alemana, en el cual se observa con frecuencia que los pacientes describen sus ataques con las expresiones "como caído del cielo" o "como un rayo". En este corpus son muy frecuentes los modificadores como de golpe y de repente.

7 Ver la entrada "como un tiro" 2. loc. adv. coloq. como una flecha <http://dle.rae.es/?id= ZrTpmUI|ZrVfwO7>. 
Si bien la descripción del dolor parte de la iniciativa del paciente, quien destaca su intensidad (línea 23: "un dolor en el pecho tremendo"), la demanda del médico por mayor precisión (línea 26) desencadena la exposición detenida de $\mathrm{P}$, a lo largo de las líneas 27 a 33: en primer lugar expresa la intensidad del dolor, mediante el cuantificador y la entonación enfática ("mUY agudo"); luego, brinda una descripción cualitativa a base de una expresión metafórica ("era era presión”, línea 29), que realiza una conceptualización habitual del dolor: EL DOLOR ES UN INSTRUMENTO OPRESIVO (para enfermedades cardíacas, Brünner y Gülich, 2002; Gallardo, 2015). Inmediatamente, $\mathrm{P}$ prosigue con la descripción, con un esforzado trabajo de formulación para precisar lo dicho (la presión aguda): "era como que me agaRRAban con una mano un pedazo de carne acá dentro así”, lo cual logra apelando a una metonimia basada en una metáfora de personificación (EL DOLOR ES UN ENTIDAD HOSTIL). El carácter subjetivo de la vivencia de la enfermedad y la dificultad de elaborar, describir y transmitir esas experiencias, explican que los pacientes recurran de manera frecuente a la metáfora y la analogía (Surmann, 2005; Lascaratou, 2007, entre otros), mecanismos que permiten vehiculizar las sensaciones y emociones de manera aproximada, por lo cual es frecuente que las expresiones metafóricas que las realizan se combinen con recursos de atenuación, entre los que se destaca por su frecuencia en este corpus la estructura "era como que" .

En las secciones siguientes me concentro en las expresiones metafóricas y las metáforas subyacentes que emplean médicos y pacientes para hablar sobre los distintos aspectos de las crisis, tratando de dilucidar si puede constatarse una selección y un empleo diferenciado según los distintos roles discursivos.

\subsection{Metáforas para el corazón}

La literatura especializada ha clasificado las metáforas de acuerdo con distintos parámetros, en general, partiendo de la clásica distinción realizada por Lakoff y Johnson (1991), quienes propusieron una tripartición entre metáforas estructurales, orientacionales y ontológicas, más tarde reformulada en términos de metáforas primarias y complejas (Lakoff y Johnson, 1999). Otro ángulo de observación es la distinción según el grado de convencionalidad o novedad, a la que se ha dedicado la literatura especializada, tanto en la tradición retórica como en la lingüística y la lexicología:

${ }^{8}$ Esta estructura y otras (también encabezadas por como) que sirven para la atenuación o la aproximación han sido estudiadas en este corpus por Ferrari (en prensa). 
así, las metáforas más convencionalizadas (como las que se presentan en unidades léxicas como célula, código), solo pueden reconocerse como tales si se recurre a la etimología; en cambio, las novedosas (como por ejemplo "las teorías son patriarcas") demandan para ser interpretadas la colaboración activa del interlocutor, quien debe "trabajar" cognitivamente para comprenderlas. Entre estos dos extremos hay evidentemente una zona de variabilidad, que se ha tratado de capturar mediante distintas denominaciones para los subtipos, como metáforas históricas, muertas, calladas, apagadas, convencionales, creativas, nuevas, etc. (cfr. Surmann, 2005: 98).

Para referirse al corazón desde la perspectiva de la salud, sobre la base de un análisis de diversos géneros escritos y orales en los que interactúan pacientes y cardiólogos (entre otros perfiles sociales), Brünner y Gülich reconstruyen el siguiente sistema de metáforas convencionales ${ }^{9}$ :

- EL CORAZÓN ES UN MOTOR (su corazón dejó de funcionar)

- EL CORAZÓN ES UNA BOMBA (es necesario que el corazón actúe bombeando la sangre hacia todos los órganos ...)

- EL Sistema de circulación CARDÍACA ES UN SISTEMA DE CAÑERÍAS O UN SISTEMA DE CALEFACCIÓN (hay que destapar una arteria)

- EL SISTEMA DE CIRCULACIÓN CARDÍACA ES UN SISTEMA DE TRÁNSITO (hubo que hacer un puente)

Resulta bastante evidente que estas conceptualizaciones tienen una amplia validez interlingüística, basada en el conocimiento especializado aceptado y vigente en cardiología, expandido a la comunicación general. Del estudio del corpus, se desprende con elocuencia que tanto médicos como pacientes recurren regularmente a expresiones metafóricas que instancian esos sistemas metafóricos. En el caso que sigue, el médico explica al paciente el evento cardíaco que ha sufrido, para lo cual emplea la metáfora convencional del sistema cardíaco concebido como un sistema de cañerías:

\section{(2) CRU 1}

1 M. ahí es donde tenía una lesión severa. digamos. o sea. es imagíneSE que las arterias son como

2 M. caÑItos como si se formara SArro adentro de la artEria y la sangre tarDAba en pasar.

3 P. CLAro

4 M. se formara SArro adentro de la artEria y la sangre tarDAba en pasar. no pasaba rápido

5 M. porque eso es lo que obsTRUye El FLUjo de SANgre [sí. entonces lo que se trató de

${ }^{9}$ Se emplean VERSALES para mencionar las metáforas conceptuales y cursivas para las expresiones metafóricas que las instancian. 
6 M. hacer es destapar esa arteria primero se destapó esa artEria y después se le pasó el

7 M. dolor bien. ahí ya vino a la unidad coroNAria y se le había ido del todo,

Pueden observarse en las líneas 1-2 que las expresiones "las arterias son como cañitos" y como si se formara sarro realizan el sistema metafórico del circuito cardíaco como sistema de cañerías: las analogías se marcan de manera explícita con los marcadores "como"y "como si"; en la línea 6 el médico -sin trabajo metalingüístico alguno- alude al procedimiento quirúrgico con la expresión "se destapó esa arteria", lo cual demuestra el alto grado de estandarización de la metáfora.

En el ejemplo 3, es el paciente quien emplea la misma metáfora (ver en línea 2 la expresión "dio que estaba tapada una arteria”) y, en la línea 4, introduce la metáfora conceptual el sistema de circulación cardíaca es un sistema de tránsito, que instancia con la expresión "era justo el desvío, una Y".

(3) CRU 3

1 P. vamos al carDIÓlogo y entonces el cardióLOgo. me hizo hacer un cateteRISmo

2 P. y el cataterismo dio que estaba tapada UNA arteria. que stent NO se podía hacer

3 M. $\mathrm{hm}$

4 P. porque era JUsto el desvío una y. entonces cuando la docTOra VIO el cateterismo

5 P. d/aparte de Ese, problema que me dice tenemos que abRIr'. porque stent no podía ser <espiración〉

El estudio de las entrevistas revela que las expresiones metafóricas convencionales para hablar del corazón son empleadas de manera regular tanto por médicos como por pacientes. Al tratarse de metáforas establecidas y difundidas, las expresiones metafóricas se emplean sin marcas o comentarios metalingüísticos (el caso del paciente en el último ejemplo, en la línea 4, que dice "era JUsto el desvío una y" es elocuente).

Sin embargo, se pueden observar algunas diferencias en la selección y en las funciones que desempeñan las expresiones metafóricas en las contribuciones de los interlocutores, que parecen vincularse con los distintos papeles discursivos.

\subsubsection{Las metáforas en las contribuciones de los médicos}

Los médicos emplean metáforas mayormente convencionales, a los fines de nombrar (conceptos especializados) y de explicar eventos, potencialmente complejos para los pacientes.

En el primer caso, como es lógico, se sirven de terminología médica, acuñada sobre la base de palabras generales, resemantizadas por creación 
metafórica (por ejemplo, arritmia silente o salva de extrasístole) ${ }^{10}$. Por otra parte es muy usual que los médicos empleen metáforas para transmitir de manera comprensible y amigable sucesos complejos a sus interlocutores: la analogía con sucesos y/u objetos del mundo cotidiano es un recurso clásico que favorece la comprensibilidad. Obsérvese el siguiente ejemplo:

\section{(4) CLT}

1 creo que la situación o el estado de abatimiento que usted tiene es el betabloquea:nte. porque

2 haciéndolo fácil el bletaboqueante es un bloqueante. no muy específico y bloquea todo. bloquea desde

3 la vigilia. bloquea la líbido. bloquea la capacidad física. bloquea la frecuencia cardíaca. todo es en

4 bA:ja es como si a uno- como que a usted lo desenchufaran y anda sin pila, sin energía eso de

5 quedarse dormido es pérdida de atención y de la concentración. todo eso son efectos secundarios del

6 betabloqueante

El médico intenta explicar la acción de los betabloqueantes que ingiere el paciente y que evidentemente le ocasionan un estado de falta de energía: su afán explicativo se explicita con un comentario metadiscursivo: en la línea 2, emplea la expresión "haciéndolo fácil” con la que inicia una secuencia didáctica, que culmina en la línea 4 con una metáfora de comparación, introducida por dos estructuras que indican cierta vaguedad o atenuación: "es como si" y "como que". La analogía con la electricidad y las baterías ("andar sin pila") permite ilustrar con claridad la acción indeseada de los medicamentos.

La presencia asidua en el corpus de este tipo de metáfora -muy convencionalizadas en general-sugiere la existencia de un budget de metáforas relativamente acotado y establecido, que ayudan al médico a transmitir conocimiento potencialmente complejo de manera amigable. En el ejemplo 5 la formulación "yo siempre digo" en la línea 3 muestra que la metáfora de comparación empleada forma parte de un repertorio rutinizado en el discurso de este médico.

\section{(5) CLT}

1 M. ya está empezando a enfermar la aurícula y la forma de enfermarla es generar fibrosis y la aurícula

2 M. se empieza a agrandar. cuanto más grande es la aurícula menos chances de poder controlar la

3 M. arritmia. yo siempre digo que esto es como controlar un casal de perros en un campito chiquito

4 M. o un casal de perros en un campo de setecientas hectáreas es muy dificil saber dónde están

5 M. con la arritmia pasa lo mismo,

${ }^{10}$ Para una presentación más detenida, cfr. Ciapuscio (en prensa). 
En este caso el agrandamiento relativo de la aurícula y su posibilidad más o menos efectiva de tratamiento se compara con la probabilidad de atrapar un casal de perros en una extensión geográfica (campito/campo): el evento cardiológico se hace accesible a partir de la correspondencia con objetos de la vida cotidiana. En síntesis, se puede afirmar que los médicos -en este corpus- emplean metáforas convencionales o rutinizadas, fundamentalmente con el fin de referir a conceptos o eventos de la disciplina y para explicar de manera accesible conocimiento especializado.

\subsubsection{Las metáforas en el discurso de los pacientes}

Como se ha visto en el ejemplo 3, los pacientes también emplean metáforas convencionales para hablar de sus problemas cardíacos, puesto que se trata de conceptualizaciones muy difundidas en la comunicación pública sobre temas médicos. Sin embargo, resulta mucho más interesante detenerse en el discurso de los pacientes cuando hablan de sus vivencias subjetivas sobre el dolor cardíaco y los síntomas concomitantes. Como lo han mostrado investigaciones previas, las personas afectadas suelen verbalizar o aludir de distintas maneras a la dificultad de transmitir aspectos subjetivos de la experiencia de la enfermedad y del dolor (Gülich y Schöndienst, 1999; Gülich y Furchner, 2002; Lascaratou, 2007; Ciapuscio, 2016). Son frecuentes en el corpus las expresiones que aluden a esas dificultades e incluso a la imposibilidad de transmitir la experiencia dolorosa, como lo ilustran las siguientes muestras del corpus:

- no. no te puedo decir porque era algo muy especial (CLR)

- pero el dolor del pecho no. nO TEngo fOrma de expliCArlo (CRU)

El carácter impreciso o difuso de las vivencias del dolor, el compromiso emocional que este causa, explica las dificultades de los pacientes para describir sus sensaciones. Por eso, como se ha afirmado antes, recurren a esquemas de formulación que incluyen expresiones metafóricas, acompañadas de abundantes recursos de atenuación y vaguedad. En lo que sigue, me detengo en las expresiones y conceptualizaciones subyacentes sobre el dolor cardíaco. En primer lugar, se presenta un cuadro en el que se sistematizan las conceptualizaciones metafóricas que subyacen a las descripciones de los pacientes del dolor cardíaco y ejemplos de enunciados que las evidencian. En segundo lugar, analizo una selección de fragmentos de dos interacciones de manera más detallada. 
CuAdro 1. Metáforas y expresiones metafóricas del dolor cardíaco

\begin{tabular}{|c|c|}
\hline Metáforas conceptuales & Expresiones metafóricas \\
\hline $\begin{array}{l}\text { EL DOLOR } \\
\text { ES UN INSTRUMENTO FILOSO }\end{array}$ & $\begin{array}{l}\text { como si hubiera ALgo: que me pinCHAra o me } \\
\text { quisiera corTAr algo así }\end{array}$ \\
\hline $\begin{array}{l}\text { EL DOLOR } \\
\text { ES UN INSTRUMENTO } \\
\text { OPRESIVO }\end{array}$ & $\begin{array}{l}\text { era algo como que me pulSAba. lo del } \\
\text { brAzo es como cuando te toman la presión. } \\
\text { que te van apreTANdo y: parece que te. } \\
\text { te DUEle MAl' bUEno. el del brazo era así. }\end{array}$ \\
\hline $\begin{array}{l}\text { EL DOLOR } \\
\text { ES UN DISPARO/GOLPE }\end{array}$ & $\begin{array}{l}\text { y de repente siento como un imPACto Seco } \\
\text { en el pEcho. (...) } \\
\text { de repentE: leVANto el balde. cuando } \\
\text { el balde siento un impacto muy fuErte } \\
\text { en el Pecho. que me tira.. } \\
\text { de repente senti un fuerte golpe en el pecho. }\end{array}$ \\
\hline EL DOLOR ES FUEGO & $\begin{array}{l}\text { BUeno. eh el/ el del pEcho no sé si } \\
\text { decIrte si era dolor. sI Era como una } \\
\text { quemazón que te agArra una ardor y te: } \\
\text { duele un poQUIto. a mí }\end{array}$ \\
\hline $\begin{array}{l}\text { EL DOLOR ES UNA ENTIDAD } \\
\text { HOSTIL }\end{array}$ & $\begin{array}{l}\text { P: era era presión era como que me agaRRAban } \\
\text { con una mano un pedazo de carne acá dentro } \\
\text { así }\end{array}$ \\
\hline
\end{tabular}

Como puede verse, los pacientes emplean expresiones metafóricas regulares para la expresión del dolor (pinchazo, ardor, quemazón, presión, cosquilleo), que suelen recoger las grillas del dolor que emplean distintas instituciones médicas (como la difundida taxonomía de la Universidad McGill). Estas expresiones realizan metáforas conceptuales que coinciden con las identificadas en otras lenguas -por ejemplo, Kövecses (2008) para el dolor en general y Brünner y Gülich (2002) para la expresión de dolor cardíaco): EL DOLOR ES UN INSTRUMENTO FILOSO, OPRESIVO, EL DOLOR ES UN DISPARO, EL DOLOR ES FUEGO, EL DOLOR ES UNA ENTIDAD HOSTIL. En general, en síntesis, el dolor cardíaco se describe como provocado por un instrumento o agente indeterminado externo (que agrede).

En el ejemplo (1) que reproduzco parcialmente aquí como (6) se evidencia la elaboración propia y original de las metáforas EL DOLOR ES PRESIÓN, EL DOLOR ES PROVOCADO POR UN AGENTE EXTERNO, que transmite con dramatismo la experiencia de dolor vivida: 
(6) CRU 1

1 M: [y el dolOR cómo era' era/'

2 P: era $m U Y$ agUdo

3 M: [qué sentía usted'

4 P: era era preSión era como que me agaRRAban con una mano un pedazo de carne

5 P: acá dentro así <ruido con la boca de desgarro>

El agente externo, causa del dolor, se formula con un plural indeterminado -"me agarraban" (línea 4) - que ejecuta la agresión con una mano ficticia. Como es frecuente, las expresiones metafóricas van precedidas de índices atenuadores que expresan la imprecisión o, como en este caso, la ficcionalidad, y el carácter aproximado ("era como que"). La expresión "un pedazo de carne" seguida de la onomatopeya que reproduce el sonido del desgarro otorga un carácter vívido y dramático a la descripción del dolor (líneas 4 y 5 ).

Los pacientes suelen presentar metáforas personales y creativas para la descripción del dolor y de los síntomas concomitantes. En el ejemplo que sigue, el paciente elabora una metáfora novedosa para dar cuenta de su particular percepción, metáfora que persiste, es reelaborada y reformulada a lo largo de toda la entrevista. El evento cardíaco sufrido se desarrolla a lo largo de varios días, en los que el paciente sufre distintos síntomas y picos de dolor, concurre a distintos puestos sanitarios, sin que se le detecten evidencias de la crisis cardíaca, hasta que finalmente ocurre el infarto, que es atendido en el hospital "El Cruce". En la entrevista el paciente relata el proceso y los distintos episodios de dolor cardíaco, para lo que reiteradamente echa mano de una conceptualización metafórica personal:

(7) CRU 2

1 M. [cómo era ese dolor'. era una punTAda' un ardor

2 P. una puntAda acá en el centro

3 M. ajá. y se le iba para la espalda el cuelló los brazos

4 P. no eh a la mISma vez era la punTAda y el ardor $\quad$ y/ yo sentía que NO=no. le explico e:

5 P. imaGInese el cuerpo. los pulmones. era como que venía del pulmóN y yo lo sentía como

6 P. que venía por aDENtro del pulmón hacia el pecho era como que Era un puentec/ e/ Era

7 P. en MI imaginacIón como yo lo sentía

$8 \mathrm{M}$ sisisi es lo que sentía

9 P. CLAro. era como que venía ahí sí: los hOMbros y: yO.. esto fue el viErnes cuando 10 M. y los brazos no le dolían' 
11 P. fui al (?upa). el MARtes no me pasó. eh: aHÍ fue cuando yo me empecé a/ a: darme cuenta

12 P. que Se me adormecía. empezaba en la clavícula hombros y brazos,.. nada más.

13 P. Y el fuEgo. el dolor y el imPACto de como que venía del pulmón un puenteCIto así. <jocoso>

14 P. yo lo describo de esa manera + porque era lo que sentía por dentro el puentecito de:/. la $15 \mathrm{M}$. perfecto

16 P. fatiga que venía del pulmón hacia el pecho

En la línea 1 el médico interroga sobre la calidad del dolor ofreciendo alternativas (puntada, ardor) que muestran el carácter regular o estandarizado de las conceptualizaciones del dolor cardíaco. El paciente ratifica la primera opción, pero ante la pregunta del médico por la expansión del dolor (línea 3), quien supone la direccionalidad habitual del pecho hacia la espalda, el cuello, los brazos, corrige su expresión anterior ("eh a la mISma vez era la punTAda y el ardor", línea 4) y niega aquella suposición. A partir de ese momento, $\mathrm{P}$ inicia un trabajo de elaboración rico en recursos, para dar cuenta de una conceptualización personal del dolor como movimiento: su dolor surge del interior del cuerpo, desde los pulmones, y llega al pecho, en un movimiento ascendente desde lo profundo hacia la superficie.

Sobre el final de la línea 4 emplea la expresión "le explico", que realiza una acción lingüística de composición textual (cfr. Gülich y Kotschi, 1987: 212), para iniciar una extensa secuencia en la que laboriosamente verbaliza su percepción; para ello, comienza por solicitar al médico que se imagine mentalmente el cuerpo y los pulmones (línea 5): a partir de allí, introduce una serie de estructuras que encabeza con el verbo ser y el marcador de atenuación y/o aproximación "como que": en primer lugar, describe el lugar de origen ("era como que venía del pulmón”), a continuación reformula la primera expresión, enmarcándola con el pronombre personal yo seguido del verbo de emoción "sentir", y expandiéndola con una precisión: "y yo lo sentía como que venía por aDENtro del pulmón hacia el pecho" (líneas 5 y 6). Inmediatamente, para ilustrar el movimiento, introduce la analogía con un puente pequeño, con la que expresa su percepción subjetiva (la metáfora nuevamente encabezada por como que: "era como que Era un puentec(ito)"); es interesante destacar que el paciente, además, comenta y justifica su metáfora en la línea 7: "Era en MI imaginacIÓn como yo lo sentía”, demostrando el control cognitivo de su propio discurso. El médico, en la línea 7, manifiesta de manera enfática su comprensión y aceptación de la metáfora empleada ("sí, sí, es lo que sentía”).

En síntesis, la percepción del dolor cardíaco se conceptualiza en términos de un esquema de TRAYECTO (Lakoff y Johnson, 1999), con un punto inicial y un punto final, en un sentido ascendente, de los pulmones hacia 
el pecho, que transmite la metáfora del "puentecito". La metáfora, como adelanté, persiste en la entrevista: en la línea $13 \mathrm{P}$ resume sus percepciones, haciendo uso de dos sistemas metafóricos convencionales (EL DOLOR ES FUEGO, EL DOLOR ES UN IMPACTO) para luego insistir con su metáfora personal: "Y el fuEgo. el dolor y el imPACto de como que venía del pulmón un puenteCIto así"; nuevamente la expresión metafórica es comentada mediante una expresión: "yo lo describo de esa manera porque era lo que sentía por dentro el puentecito de la fatiga".

Un poco más avanzada la conversación con el cardiólogo, el paciente narra un segundo episodio de su crisis cardíaca y reformula la metáfora del puente:

1 M: el dolor [era muy fuerte'

2 P: \& CLAro. era muy inTENso y seguÍa sintiendo ese caminito del pulmón hacia el PEcho

Puede observarse en la línea 2 la secuencia reformulativa, compuesta por una construcción formada por el pronombre demostrativo "ese", que posibilita la evocación por parte del interlocutor de la expresión fuente de la reformulación, el puentecito -ya bastante lejana en el discurso- seguida de una segunda expresión metafórica ("caminito"), que expresa nuevamente la percepción del dolor como un movimiento de trayecto interiorexterior: de un punto de partida (el pulmón) a uno de llegada (pecho).

Finalmente, ya en los tramos finales de la entrevista, llega a la descripción del suceso crítico central, que se desencadena tres días más tarde, y que lo lleva a la hospitalización:
1 P. a las seis de la mañana . seis golpes
tuve. sei imPACto. fuERtes
$2 \mathrm{M}$.
cuando usted dice golpes es como que el dolor es muy fuerte
3 P. que me sentaron en la cama y e/ y el el dolor MUY fuerte
4 P. y YO me lo calmaba aCÁ.. me agarraba acá y me hacía fuerte acá en una. me masajeaba porque
5 P. el dolor era como que lo tenía aCÁ tremENdo y seguía el supuesto puentecito entre la columna
6 P. y:: BAh los pulmone entonce digo. la PUta madre esto. Esto es de los pulMOnes. si NO me
7 P. encontraron en el corazón. ESto tiene que ser de los pulmones,

La expresión metafórica en la línea 5 -“el supuesto puentecito”-, que reformula las menciones previas, también incorpora un marcador atenuativo de distancia (el adjetivo "supuesto") que señala nuevamente la regulación metadiscursiva y el monitoreo del narrador sobre su propio discurso. 


\subsubsection{Metáforas en el trabajo interactivo}

El último caso que deseo comentar es muy interesante, puesto que muestra el trabajo interactivo del paciente y del médico, centrado en la descripción del síntoma y la elaboración del diagnóstico, a base de una metáfora creativa propuesta por el paciente.

Como en los casos ya comentados, el paciente explicita y tematiza el carácter aproximado y tentativo de sus percepciones, que se expresa en un arduo trabajo de formulación. En (10) se presentan los fragmentos iniciales de la entrevista, en los que el paciente introduce la metáfora de la burbuja de aire para verbalizar y transmitir sus sensaciones:

\section{(10) CLT}

1 M. buEno... buEno y <rápido > cómo vamos' cómo vamos'+ co/cómo fue todo' CUEnte

2 P. NO. anduve bIEn pero e: lo que tengo. e ya me ha pasado DOS veces. y la última fue: ahora el

3 P. sábado es como que se me forma una burbuja de AIre=acÁ y yo elimino erucTANdo

$4 \mathrm{M}$.

eructando

5 P. pero que no tiene no nada que ver con lo estomaCAL. no con la comida (...)

6 P. TEngo un dolor acÁ. e/es como que crece al

como si el PEcho no tuviese esPAcio

$7 \mathrm{M}$.

son como espasmos

8 P. para contener el aire que se forma y ahi me/ e. no es que sea un dolor insoportable pero SIENto. 9 M. no. pero

10 P. dolor un poco. eLIMIno. el

$11 \mathrm{M}$.

SI <rápido > se va con eso + ahora por qué' se

12 P. forma es/esa como burbuja de aire ahi no sé,... es lo Único que puedo decir que SIENto

En la línea 3, en el marco de una narración iterativa ("me ha pasado dos veces"), P introduce la metáfora de la burbuja (que se convertirá en tema principal de la conversación con el médico), en el marco de una proposición condicional encabezada por "como que" y la deixis al cuerpo (el pecho); más abajo, en las líneas 6 y 8 , desarrolla y explica esa metáfora a través de un intenso trabajo reformulativo: "es como que crece/como si el pecho no tuviese espacio para contener el aire que se forma”. La reiteración de las construcciones con "como" y el empleo de proposiciones condicionales hipotéticas (como si) transmiten el carácter aproximado y tentativo de las descripciones; en las líneas 10-12 se interroga por la causa de ese malestar y retoma la metáfora conceptual con el sintagma nominal en- 
cabezado por el pronombre demostrativo ("es/esa como burbuja de aire ahí"), en la que sin embargo intercala nuevamente el nexo "como", reiterando así el carácter aproximativo de la metáfora empleada y su explícito distanciamiento. En la línea 7, el médico propone una primera interpretación ("son como espasmos"), iniciando así, en colaboración con el paciente, un extenso trabajo de construcción de hipótesis tendientes al diagnóstico, basado en la metáfora de la burbuja, que se extenderá a lo largo de la entrevista.

En tanto que el paciente realiza su metáfora con formulaciones que -en todos los casos- contienen atenuadores y construcciones hipotéticas, el médico propone alternativas conceptuales bajo la forma de reformulaciones sucesivas, en las que predomina el carácter asertivo, el cual se instancia en el modo verbal de las expresiones predicativas, en marcadores modales de certeza y en expresiones referenciales conformadas como descripciones definidas. A continuación, algunos fragmentos de la entrevista para ilustrar las intervenciones del médico:

1 M. MÁS si es con eRUctos porque es una cuestión digesTIva=en realidad es retención de aire

2 P. $\quad \mathrm{m}: \ldots$ si pero no/no se vinCUlan digamos

3 P. con comidas por ahí se geNEra'. muy LEjos de las comidas

4 M. no=no=no (? lo digestivo alarga) $\mathrm{mhm} \quad$ si $=\mathrm{si}$

5 M. no necesariamente las coMIdas. la prioridad este: e: en geneRAL la causa. fundamental

6 M. por la que uno tiene: GAses no es tanto la coMIda como que TRAga aire eso/ESA es la razón

7 M. más importante por la que uno: acuMUla aire e incluso se producen. espasmos esofágicos no

8 M. TANto por el tipo de comida.. que por el tipo de comida: si: se producen GAses pero son MAS.

9 M. intestinAles digamos como: en un metabolismo

10 P. a ver. pero digo. localizado ACA'.. n/no baja de ahí sino es de ACA'

11 P. y de ACA' (?... si:

12 M. eRucta y: y eso le produce la opresión, <rápido $>$ si. no tiene nada que ver con el procedimiento

13 M. a esta altura yo creo que es una aerofagia ahora quE ES': este/que está/que TRAga Aire y a

14 M. partir de ese momento: la burbuja: la tiene que expulsar y lógico en el esófago eso es molesto (...)

15 M. PEro es un: TEma ese porque: esas: esas burbujas y/esos espasmos de/esofágicos también

16 M. generan cier/no se si se acuerda que: que le expliqué que había una relación: 
17 M. SIN queRER uno inconsciENtemente. traga aire: 〈rápido> esa es una cosa muy frecuente:'+ y

18 M. eso hay que expulSARlo [. y porque está bien alto lo expulsa. porque la burbuja ocupa lugar'

19 M. por eso molesta uno eructa y se acabó, en el ACto se va, [no'

$20 \mathrm{P}$.

sí=sí=sí

Como puede verse en la transcripción, el médico "trabaja” en colaboración con el paciente para ir delineando y determinando el diagnóstico y su posible relación con la arritmia cardíaca sobre la base de la metáfora de la burbuja. Ese trabajo y esfuerzo cognitivo y de formulación le permite avanzar en la comprensión y clarificación del síntoma, hasta finalmente estar en condiciones de denominarlo de manera precisa; a continuación, las expresiones del médico que muestran el proceso de construcción:

- $\quad$ (son como) espasmos

- (es) retención de aire

- (son) espasmos esofágicos

- esos espasmos

- aerofagia

La conclusión diagnóstica, como es propio del discurso especializado, se formula con la modalidad hipotética ("yo creo que es una aerofagia", en la línea 13). El médico lleva a cabo ese trabajo cognitivo y formulativo reelaborando y expandiendo la conceptualización metafórica del paciente ("la burbuja"); sin embargo, para referirse a ella emplea esquemas de formulación sensiblemente diferentes de los utilizados por el paciente. En efecto, las menciones a la metáfora, en todos los casos, se realizan en términos de descripciones definidas, precedidas de los artículos o del pronombre demostrativo; en el ejemplo 10: "la burbuja" (líneas 14 y 18) y "esas burbujas" (línea 15). Podría hablarse, en este caso, de un caso de transferencia: la metáfora conceptual de la burbuja empleada con distanciamiento mediante formulaciones aproximativas e hipotéticas por el paciente es adoptada sin reservas ni distanciamiento por el médico, lo cual se revela en formulaciones declarativas y descripciones definidas, a tal punto que incluso llega a producir coordinaciones en las que equipara la expresión metafórica a la descripción médica; ver la línea 15: "esas: esas burbujas y/esos espasmos de/esofágicos”. De este modo el cardiólogo hace suya la metáfora del paciente y la aprovecha de manera inteligente para focalizar, describir y explicar el fenómeno médico.

Con este último ejemplo se ha tratado de ilustrar el trabajo interactivo y colaborativo que realizan el paciente y el médico para llevar adelante sus tareas conversacionales y alcanzar las metas discursivas; en este caso, obte- 
ner y elaborar información vital para el diagnóstico y el tratamiento. Los interlocutores, a partir de las percepciones subjetivas del dolor o malestar, conceptualizadas y verbalizadas con recurso a la metáfora, en primer lugar, las convierten en objeto central de la conversación, y luego, en colaboración, las elaboran y profundizan, para lograr la construcción del diagnóstico y definir el tratamiento.

\section{Conclusiones}

En este trabajo se ha tratado, en primer lugar, de describir e ilustrar cómo los pacientes reconstruyen y describen en colaboración con los cardiólogos -y en ocasiones con familiares- episodios de crisis cardíacas y sucesos vinculados con ellas (antecedentes de la enfermedad, sucesos concomitantes, síntomas y dolencias). El análisis de los ejemplos ha mostrado que los interlocutores, para llevar a cabo la tarea conversacional, recurren de manera regular a distintas técnicas narrativas -la narración episódica, iterativa y la escenificación dramática- en las que tienen un papel sobresaliente los procedimientos de formulación, especialmente, los metafóricos. En segundo lugar, se ha hecho foco en las metáforas que usan los interlocutores: el estudio del corpus reveló que médicos y pacientes recurren asiduamente al instrumento metafórico, pero que realizan una selección y un empleo diferenciado del tipo de metáforas, motivados en las distintas funciones y necesidades comunicativas que distinguen a sus respectivos roles.

En el discurso de los médicos prevalecen metáforas con alto grado de convencionalidad que se emplean mayormente para referir a aspectos de la enfermedad (terminología médica) y para explicar eventos complejos a los pacientes (especialmente bajo la forma de analogías). Asimismo, para transmitir conocimientos relativos al funcionamiento del sistema cardíaco y a las enfermedades asociadas con él, se valen de metáforas convencionales (como por ejemplo, el circuito cardíaco es un sistema de cañerías / un sistema de tránsito), extendidas ampliamente al campo de la comunicación pública. Los pacientes emplean ocasionalmente estas metáforas convencionales para hablar de aspectos técnicos de la enfermedad, que han incorporado a su acervo léxico a partir de su particular "experticia" (Brünner y Gülich, 2002), lograda por su carácter de afectados o por su formación general. En ambos casos, desde el punto de vista del trabajo de formulación, el empleo de expresiones metafóricas convencionales tiene su correlato en el discurso (cfr. Bregant, 2015): tales expresiones se emplean generalmente sin señales o comentarios metalingüísticos que indiquen su carácter metafórico, debido justamente al amplio conocimiento y extensión que tienen en la comunicación general. 
En el artículo se han reconstruido y descrito las conceptualizaciones metafóricas del dolor y de los síntomas de crisis cardíacas que subyacen a las contribuciones de los pacientes, sobre la base de las expresiones metafóricas relevadas: se ha constatado que para describir sus dolores recurren a metáforas que revelan cierta convencionalidad, en la medida que coinciden con las taxonomías del dolor empleadas por instituciones médicas y, también, con los resultados de investigaciones lingüísticas sobre la expresión del dolor en distintas lenguas naturales, como por ejemplo: EL DOLOR ES UN INSTRUMENTO FILOSO, EL DOLOR ES PRESIÓN o EL DOLOR ES UNA ENTIDAD HOSTIL (Kövecses, 2000; Brünner y Gülich, 2002; Surmann, 2005; Laskaratou, 2007; Ciapuscio, 2016, entre otros). Sin embargo, en sus contribuciones se destaca la presencia de metáforas nuevas y creativas, que elaboran y desarrollan para describir de manera más precisa los síntomas y el dolor experimentado durante la enfermedad. La dificultad de transmitir el dolor cardíaco y sus síntomas encuentra su vehículo en el recurso a la conceptualización metafórica personal (la mano que agarra, el puentecito, el caminito, la burbuja). La verbalización del dolor y de los distintos síntomas mediante metáforas nuevas que intentan describirlos trae aparejado un profuso trabajo de formulación por parte de los pacientes, quienes emplean un variado repertorio de procedimientos de tratamiento, como reformulaciones y comentarios, y calificaciones metadiscursivas, que se instancian a base de diferentes recursos lingüísticos, esencialmente pronombres neutros y sustantivos generales (como algo, esta cosa), construcciones hipotéticas y comparativas con atenuadores, en las que se destaca el aproximativo como que, el cual, habida cuenta de su presencia profusa en el corpus, podría considerarse un modelo de formulación preformado (Gülich, 2007), característico del discurso de los pacientes. Por lo demás, también se ha mostrado que pacientes y médicos pueden trabajar en colaboración, elaborando y precisando conceptualizaciones metafóricas novedosas, que inicialmente describen percepciones subjetivas y sensoriales del afectado -la burbuja-, que luego, sobre la base de la interacción con el especialista, pueden reformularse en un diagnóstico específico. En todo caso, el análisis aquí realizado permite ratificar la conclusión de Surmann (2005) en su trabajo sobre la metáfora en el discurso de personas con enfermedades convulsivas: las "metáforas proporcionan una salida al inconveniente de la indescriptibilidad" (ibidem: 116), es decir, de la dificultad o imposibilidad de describir con palabras los síntomas y sentimientos que experimentan los pacientes a raíz de su mal.

Finalmente, cabría quizás preguntarse si estas conceptualizaciones metafóricas de los pacientes en términos de personificaciones, ontologizaciones (instrumentos $\mathrm{u}$ objetos) o de esquemas de imagen de TRAYECTO podrían tener relevancia para el diagnóstico o el tratamiento específico, como ha 
sido constatado para el caso de las enfermedades epilépticas por parte de equipos alemanes e ingleses (Schwabe et al., 2007 y 2008). En el presente contexto, es una pregunta que queda necesariamente abierta, puesto que responderla evidentemente exigiría investigaciones más complejas y, sin lugar a dudas, interdisciplinarias. Parece razonable pensar que quizás podría ser de utilidad considerar las investigaciones del análisis del discurso sobre las narraciones de pacientes -que deberían basarse en corpus más amplios- en los importantes proyectos en curso sobre el dolor en el campo de las neurociencias, teniendo en cuenta, además, las bases neurológicas que sustentan la teoría de la metáfora contemporánea (Lakoff, 2014). 


\section{BIBLIOGRAFÍA}

ANTOS, G. (1982): Grundlagen einer Theorie des Formulierens: Textherstellung in geschriebener und gesprochener Sprache, Niemeyer, Tübingen.

Bergmann, J. y Luckmann, Th. (1995): "Reconstructive Genres of Everyday Communication", en U. Quasthoff (ed.), Aspects of Oral Communication, Berlin, de Gruyter, págs. 289-305.

BREganT, L. (2016): "Procedimientos discursivos y metáforas de diferentes grados de convencionalidad: reformulación y calificación metadiscursiva”, ponencia presentada en el XV Congreso de la Sociedad Argentina de Lingüistica, Bahía Blanca, 11-14 de mayo de 2016.

BRÜNNER, G. y Gülich, E. (2002): "Verfahren der Veranschaulichung in der Experten-Laien-Kommunikation”, en G. Brünner y E. Gülich (eds.), Krankheit verstehen. Interdisziplinäre Beiträge zur Sprache in Krankheitsdarstellungen, Bielefeld, Aisthesis Verlag, págs. 17-93.

CiAPUSCIO, G. (2003): "Formulation and reformulation procedures in verbal interactions between experts and (semi-) laypersons", Discourse Studies 5 (2), págs. 207-233.

CiAPuscio, G. (2007): “Acciones de calificación en conferencias de divulgación científica”, Revista Internacional de Lingüistica Iberoamericana (RILI), V, 1 (9), págs. 59-77.

Ciapuscio, G. (2016): "Conceptualizaciones metafóricas y recursos de formulación en narraciones de pacientes con migraña”, Oralia, 19, págs. 39-60.

Ciapuscio, G. (en prensa): "Metáforas del corazón en conversaciones sobre crisis cardíacas", en A. Cancellier, A. Cassani y E. Dal Maso (eds.), "El corazón es centro”. Narraciones, representaciones y metáforas del corazón en el mundo hispánico, Padova, Editorial Cleup.

Deignan, A. (2008): "Corpus Linguistics and Metaphor", en R. Gibbs (ed.), The Cambridge Handbook of Metaphor and Thought, Cambridge University Press, págs. 280-294.

DíAz Vera, J. (2012): "When pain is not a place: Pain and its metaphors in late middle English medical texts”, Onomázein, 26 (2), págs. 279-308.

FERRARI, L. (en prensa): "La dificultad de verbalizar el dolor cardíaco: algunas marcas léxicas", en A. Cancellier, A. Cassani y E. Dal Maso (eds.), "El corazón es centro". Narraciones, representaciones y metáforas del corazón en el mundo hispánico, Padova, Editorial Cleup.

Gallardo, S. (2015): "Metáforas que conceptualizan el dolor", Ponencia presentada en el XI Congreso Internacional de la Asociación Latinoamericana de Estudios del Discurso (ALED), Buenos Aires, octubre de 2015.

GüLICH, E. (2003): "Conversational techniques used in transferring knowledge between medical experts and non-experts”, Discourse Studies 5 (2), págs. 235263. 
GÜLICH, E. (2005): "Unbeschreibbarkeit: Rhetorischer Topos - Gattungsmerkmal - Formulierungsressource”, Gesprächsforschung - Online-Zeitschrift zur verbalen Interaktion, 6, págs. 222-244 (<www.gespraechsforschung-ozs.de >).

GülıCH, E. (2007): “'Volle Palette in Flammen'. Zur Orientierung an vorgeformten Strukturen beim Reden über Angst”, Psychotherapie \& Sozialwissenschaft 1, págs. 59-87.

Gülich, E. y Kotschi, Th. (1987): "Reformulierungshandlungen als Mittel der Textkonstitution. Untersuchungen zu französischen Texten aus mündlicher Kommunikation”, en W. Motsch (ed.), Studia Grammatica XXV, Berlin, Akademie Verlag, págs. 199-267.

Gülich, E. y Kotschi, Th. (1995): "Discourse production in oral communication”, en U. Quasthoff (ed.), Aspects of oral communication, Berlin, Mouton de Gruyter, págs. 30-66.

GüLICH E. y ScHÖNDIENST, M. (1999): “'Das ist unheimlich schwer zu beschreiben'. Formulierungsmuster in Krankheitsbeschreibungen anfallskranker Patienten: differential diagnostische und therapeutische Aspekte", Psychotherapie und Sozialwissenschaft 1, págs. 199-227.

GÜLICH, E. y FuRCHNER, I. (2002): "Die Beschreibung von Unbeschreibbarem. Eine konversationsanalytische Annäherung an Gespräche mit Anfallskranken”, en I. Keim y W. Schütte (eds.), Soziale Welten und kommunikative Stile, Tübingen, Narr, págs. 161-186.

GÜLICH, E., SchÖNDIENST, M. y SuRMANN, V. (2003): "Schmerzen erzählen Geschichten - Geschichten erzählen Schmerzen”, Psychotherapie und Sozialwissenschaft 3, V \& R, Göttingen, págs. 219-248.

HallidaY, M. A. K. (1998): "On the grammar of pain", Functions of Language 5, 1, págs. 1-32.

Kövecses, Z. (2000): Metaphor and Emotion. Language, Culture, and Body in Human Feeling, Cambridge, Cambridge University Press.

Kövecses, Z. (2008): "The conceptual structure of happiness and pain", en C. Lascaratou, A. Despotopoulou y E. Ifantidou (eds.), Reconstructing Pain and Joy: Linguistic, Literary and Cultural Perspectives, Cambridge, Cambridge Scholars Publishing, págs. 17-33.

Kövecses, Z. (2015): Where metaphors come from. Reconsidering context in metaphor. Oxford/New York, Oxford University Press.

LAKOFF, G. y JoHnson, M. (1991): Metáforas de la vida cotidiana, Madrid, Cátedra (1 $1^{\mathrm{a}}$. ed. 1980).

Lakoff, G. y Johnson, M. (1999): Philosophy in the Flesh. The embodied Mind and its Challenge to Western Thought, Basic Books, Perseus Books Group.

LAKoff, G. (2008): "The neural theory of metaphor", en R. Gibbs (ed.), The Cambridge Handbook of Metaphor and Thought, New York, Cambridge University Press, págs. 17-38.

LAKOFF, G. (2014): "Mapping the brain's metaphor circuitry: metaphorical thought in everyday reason", Frontiers in Humanneuroscience 8, disponible en: <http://journal.frontiersin.org/article/10.3389/fnhum.2014.00958/full>.

LASCARATOU, C. (2007): The language of pain, Amsterdam/Philadelphia, John Benjamins. 
REDDY, M. J. (1979): "The conduit metaphor: a case of frame conflict in our language about language", en A. Ortony (ed.), Metaphor and Thought, Cambridge, Cambridge University Press, págs. 284-310.

SEmino, E. (2010): "Descriptions of pain, metaphor and embodied simulation", Metaphor and Symbol, 25 (4), págs. 205-226.

Schwabe, M., Reuber, M., Schöndienst, M. y Gülich, E. (2008): "Listening to people with seizures: How can linguistic analysis help in the differential diagnosis of seizure disorders?”, Communication and Medicine 5 (1), págs. 59-72.

Schwabe, M., Howell, S. y Reuber, M. (2007): "Differential diagnosis of seizure disorders: A conversation analytic approach”, Social Science and Medicine, 64, págs. 712-724.

Surmann, V. (2005): Anfallsbilder. Metaphorische Konzepte im Sprechen anfallskranker Menschen, Würzburg, Königshausen \& Neumann.

Williams Camus, J. (2009): "Metaphors of cancer in scientific popularization articles in the British press", Discourse Studies 11 (4), págs. 465-495.

Williams Camus, J. (2016): "Get the metaphor right! Cancer treatment metaphors in the English and Spanish press”, Alfinge, 28, págs. 109-138. 


\section{APÉNDICE}

CONVENCIONES DE TRANSCRIPCIÓN

\begin{tabular}{|l|l|}
\hline, & Curva de entonación ascendente \\
\hline, & Curva de entonación descendente \\
\hline MAYÚSCULAS & Énfasis \\
\hline$:$ & Alargamiento de un sonido / una sílaba \\
\hline$/$ & Interrupción perceptible, corrección \\
\hline$=$ & Unión perceptible \\
\hline$*$ & Emisiones superpuestas \\
\hline$\&$ & Encabalgamiento rápido de intervenciones \\
\hline$\cdot \ldots \quad \ldots$ & Pausa : muy corta, corta, más extensa \\
\hline$(?$ palabra $)$ & Transcripción insegura \\
\hline$<$ Comentario $>+$ & El comentario es válido para el segmento hasta el signo + \\
\hline
\end{tabular}

